\title{
Editorial
}

\section{The LOFAR Two Meter Sky Survey}

The $150 \mathrm{MHz}$ channel of the LOFAR low-frequency radio telescope is being used to perform the LOFAR Two-meter Sky Survey (LoTSS), a two-tier survey that will both cover the full Northern Hemisphere to an 100 microjansky depth and observe 50 square degrees with an order of magnitude deeper sensitivity. This special issue describes the first data release of the LoTSS Deep Fields observations, along with selected early results. The newly released images cover a depth of $\sim 30$ microjanskys and $\sim 30$ square degrees in three deep fields, which were selected for their wealth of available multiwavelength data: the Lockman Hole, Boötes, and ELAIS-N1 fields. The release includes an extracted catalog of 150000 sources, half of which are associated with an optical counterpart and come with ancillary information such as photometric redshifts and inferred stellar masses. The early results of these deep field observations span a wide range of extragalactic topics, but also include a time-domain stellar physics investigation. They represent a small sample of what is yet to come, hinting at the great potential of the full LoTSS Survey.

Françoise Combes, David Elbaz, Thierry Forveille, Rubina Kotak, Laura Pentericci, Steve Shore Astronomy \& Astrophysics Editors 